\title{
Effect of Partially Distributed heat supply on an Inverse Thermoelastic Problem of Heat Conduction in an Annular Disc Due to Internal Heat Generation
}

\author{
Chandrashekhar S. Sutar \\ Assistant professor, Department of applied science, R.C.Patel institute of technology shirpur, (M.S.) India
}

Abstract: This paper consist inverse thermoelastic problem of heat conduction with the application of partially distributed heat supply and internal heat generation. In this paper unknown temperature, displacement stress functions are determined by using finite Marchi-Zgrablich transform and Fourier cosine transform. The results are obtained in the form of Bessel's function and infinite series.

Keywords: Inverse thermoelastic problem, Stress function, Thermoelastic displacement

\section{Introduction}

Roychaudhari S.K.[5] has discussed the thermal stresses in thin circular plate. Khobragade N.W. and Lamba N.K.[1] have investigated the thermal stresses of thin annular disc due to partially distributed heat supply where they have determined the stress functions, displacement function and temperature. This paper deals with the determination of thermoelastic displacement, stress functions, unknown temperature with the effect of partially distributed heat supply and internal heat generation occupying the space D: $a \leq r \leq b, 0 \leq z \leq h$. The finite Marchi-Zgrablich transform and Fourier cosine transform is used to find the results. The results are obtained in the form of infinite series and Bessel's function.

\section{Statement Of The Problem}

Consider a thin annular isotropic disc of thickness h occupying the space D: $a \leq r \leq b, 0 \leq z \leq h$. The differential equation governing the displacement function $U(r, z, t)$ as

$\frac{\partial^{2} U}{\partial r^{2}}+\frac{1}{r} \frac{\partial U}{\partial r}=(1+v) a_{t}$

With $U_{r}=0$ at $r=a$ and $r=b, v$ and $a_{t}$ are the Poisson's ratio and the linear coefficient of the thermal expansion of the material of the disc respectively and $T(r, z, t)$ is the temperature of the disc satisfying the differential equation

$\frac{\partial^{2} T}{\partial r^{2}}+\frac{1}{r} \frac{\partial T}{\partial r}+\frac{\partial^{2} T}{\partial z^{2}}+\frac{g(r, z, t)}{k}=\frac{1}{\alpha} \frac{\partial T}{\partial t}$

Where $k$ and $\alpha$ thermal conductivity and thermal diffusivity of the material of the disc subject to the initial conditions

$T(r, z, 0)=0$

The boundary conditions and interior condition are

$$
\begin{aligned}
& {\left[T+\mathrm{k}_{1} \frac{\partial T(r, z, t)}{\partial r}\right]_{r=\xi}=\frac{-Q_{0}}{\lambda} f(\xi, z, t)(\text { known })} \\
& {\left[T+\mathrm{k}_{2} \frac{\partial T(r, z, t)}{\partial r}\right]_{r=a}=F_{1}(z, t)} \\
& {\left[\frac{\partial T}{\partial z}\right]_{z=h}=F_{2}(r, t)} \\
& {\left[\frac{\partial T}{\partial z}\right]_{z=0}=F_{3}(r, t)}
\end{aligned}
$$

$[T(r, z, t)]_{r=b}=G(z, t)$ (Unknown) 
The stress functions $\sigma_{r r}$ and $\sigma_{\theta \theta}$ are given by

$\sigma_{r r}=-2 \mu \frac{1}{r} \frac{\partial U}{\partial r}$

$\sigma_{\theta \theta}=-2 \mu \frac{\partial^{2} U}{\partial r^{2}}$

Where $\mu$ is the Lame's constant, while each of stress function $\sigma_{r z}, \sigma_{z z}, \sigma_{\theta z}$ are zero within the disc in the plane state of stress.

The equations (1) to (10) constitute the mathematical formulation of the problem under consideration.

\section{Solution Of The Problem}

The finite Marchi-Zgrablich integral transform of order $p$ is defined as

$\bar{f}_{p}(n)=\int_{a}^{b} x f(x) S_{p}\left(\mathrm{k}_{1}, \mathrm{k}_{2}, \mu_{\mathrm{n}} x\right) d x$

And inverse Marchi-Zgrablich integral transform as

$f(x)=\sum_{n=1}^{\infty} \frac{\bar{f}_{p}(n) S_{p}\left(\mathrm{k}_{1}, \mathrm{k}_{2}, \mu_{\mathrm{n}} x\right)}{c_{n}}$

Where

$$
\begin{gathered}
S_{p}\left(\mathrm{k}_{1}, \mathrm{k}_{2}, \mu_{\mathrm{n}} x\right)=J_{p}\left(\mu_{\mathrm{n}} x\right)\left\{G_{p}\left(\mathrm{k}_{1}, \mu_{\mathrm{n}} a\right)+G_{p}\left(\mathrm{k}_{2}, \mu_{\mathrm{n}} b\right)\right\}-G_{p}\left(\mu_{\mathrm{n}} x\right)\left\{J_{p}\left(\mathrm{k}_{1}, \mu_{\mathrm{n}} a\right)+J_{p}\left(\mathrm{k}_{2}, \mu_{\mathrm{n}} b\right)\right\} \\
c_{n}=\frac{b^{2}}{2}\left\{S_{p}{ }^{2}\left(\mathrm{k}_{1}, \mathrm{k}_{2}, \mu_{\mathrm{n}} b\right)-S_{p-1}\left(\mathrm{k}_{1}, \mathrm{k}_{2}, \mu_{\mathrm{n}} b\right) \cdot S_{p+1}\left(\mathrm{k}_{1}, \mathrm{k}_{2}, \mu_{\mathrm{n}} b\right)\right\}- \\
\frac{a^{2}}{2}\left\{S_{p}{ }^{2}\left(\mathrm{k}_{1}, \mathrm{k}_{2}, \mu_{\mathrm{n}} a\right)-S_{p-1}\left(\mathrm{k}_{1}, \mathrm{k}_{2}, \mu_{\mathrm{n}} a\right) \cdot S_{p+1}\left(\mathrm{k}_{1}, \mathrm{k}_{2}, \mu_{\mathrm{n}} a\right)\right\}
\end{gathered}
$$

An operational property is given by

$\int_{a}^{b}\left[\frac{\partial^{2} f}{\partial x^{2}}+\frac{1}{x} \frac{\partial f}{\partial x}+\frac{p^{2} f}{x^{2}}\right] S_{p}{ }^{2}\left(\mathrm{k}_{1}, \mathrm{k}_{2}, \mu_{\mathrm{n}} x\right)=\frac{b}{\mathrm{k}_{2}} S_{p}{ }^{2}\left(\mathrm{k}_{1}, \mathrm{k}_{2}, \mu_{\mathrm{n}} b\right)\left[f+\mathrm{k}_{2} \frac{\partial f}{\partial x}\right]_{x=b}-\frac{a}{\mathrm{k}_{1}} S_{p}{ }^{2}\left(\mathrm{k}_{1}, \mathrm{k}_{2}, \mu_{\mathrm{n}} a\right)[f+$ $\mathrm{k} 1 \partial f \partial x x=a-\mu \mathrm{n} 2 f p n$

(13)

If $f(x)$ satisfies Dirichelet's condition in the interval $(0, a)$ and if for that range its finite Fourier Cosine transform is defined to be

$\bar{f}_{c}(l)=\int_{0}^{a} f(x) \cos \frac{l \pi x}{a} d x$

Then at each point $(0, a)$ at which $\mathrm{f}(\mathrm{x})$ is continuous, Inverse finite Fourier Cosine transform is given by

$f(x)=\frac{\bar{f}_{c}(0)}{a}+\frac{2}{a} \sum_{l=1}^{\infty} \bar{f}_{c}(l) \cos \frac{l \pi x}{a}$

Applying Finite Marchi-Zgrablich transform stated in (11) to (13) to the equations (2),(3),(6) and (7) and using equations (4), (5), also again applying Finite Fourier Cosine transform as in (14), using given boundary conditions and then taking their inverses it gets

$$
\begin{aligned}
& \mathrm{T}=\frac{-2 \alpha}{\mathrm{h}} \sum_{\mathrm{m}=1}^{\infty}\left\{\sum _ { \mathrm { n } = 1 } ^ { \infty } \mathrm { e } ^ { - \alpha ( \mu _ { \mathrm { m } } ^ { 2 } + \frac { \mathrm { n } ^ { 2 } \pi ^ { 2 } } { \mathrm { h } ^ { 2 } } ) t } \left[\int \left\langle\frac{\xi}{\mathrm{k}_{1}} \mathrm{~s}_{0}^{2}\left(\mathrm{k}_{1}, \mathrm{k}_{2}, \mu_{\mathrm{m}} \xi\right) \frac{\mathrm{Q}_{0}}{\lambda} \mathrm{f}^{*}+\frac{\mathrm{a}}{\mathrm{k}_{2}} \mathrm{~s}_{0}^{2}\left(\mathrm{k}_{1}, \mathrm{k}_{2}, \mu_{\mathrm{m}} \mathrm{a}\right) \mathrm{F}_{1}^{*}-(-1)^{\mathrm{n}} \overline{\mathrm{F}_{2}}+\right.\right.\right. \\
& \left.\left.\left.\overline{\mathrm{F}_{3}}-\frac{\overline{\mathrm{g}}^{*}}{\mathrm{k}}\right\rangle \mathrm{e}^{\alpha\left(\mu_{\mathrm{m}}^{2}+\frac{\mathrm{n}^{2} \pi^{2}}{\mathrm{~h}^{2}}\right) \mathrm{t}} \mathrm{dt}-\frac{\mathrm{T}_{0}}{\alpha}\right] \cos \left(\frac{\mathrm{n} \pi \mathrm{z}}{\mathrm{h}}\right)+\frac{\emptyset}{2}\right\} \frac{\mathrm{s}_{0}\left(\mathrm{k}_{1}, \mathrm{k}_{2}, \mu_{\mathrm{m}} \mathrm{r}\right)}{\mathrm{c}_{\mathrm{m}}}
\end{aligned}
$$

Where 
$\emptyset=$

$\left\{e^{-\alpha\left(\mu_{m}^{2}+\frac{n^{2} \pi^{2}}{h^{2}}\right) t}\left[\int\left\langle\frac{\xi}{k_{1}} s_{0}^{2}\left(k_{1}, k_{2}, \mu_{m} \xi\right) \frac{Q_{0}}{\lambda} f^{*}+\frac{a}{k_{2}} s_{0}^{2}\left(k_{1}, k_{2}, \mu_{m} a\right) F_{1}^{*}-(-1)^{n} \overline{F_{2}}+\overline{F_{3}}-\frac{\bar{g}^{*}}{k}\right\rangle e^{\alpha\left(\mu_{m}^{2}+\frac{n^{2} \pi^{2}}{h^{2}}\right) t} d t-\right.\right.$

TOan=0

AND

$T_{0}=\left[\alpha \int\left\langle\frac{\xi}{k_{1}} s_{0}^{2}\left(k_{1}, k_{2}, \mu_{m} \xi\right) \frac{Q_{0}}{\lambda} f^{*}+\frac{a}{k_{2}} s_{0}^{2}\left(k_{1}, k_{2}, \mu_{m} a\right) F_{1}^{*}-(-1)^{n} \overline{F_{2}}+\overline{F_{3}}-\frac{\bar{g}^{*}}{k}\right\rangle e^{\alpha\left(\mu_{m}^{2}+\frac{n^{2} \pi^{2}}{h^{2}}\right) t} d t\right]_{t=0}$

Also - and $*$ denotes the Finite Marchi-Zgrablich transform and Finite Fourier Cosine transform respectively.

Now put $r=b$ in (16) it gets

$G=\frac{-2 \alpha}{h} \sum_{m=1}^{\infty}\left\{\sum_{n=1}^{\infty} e^{-\alpha\left(\mu_{m}^{2}+\frac{n^{2} \pi^{2}}{h^{2}}\right) t}\left[\int\left\langle\frac{\xi}{k_{1}} s_{0}^{2}\left(k_{1}, k_{2}, \mu_{m} \xi\right) \frac{Q_{0}}{\lambda} f^{*}+\frac{a}{k_{2}} s_{0}^{2}\left(k_{1}, k_{2}, \mu_{m} a\right) F_{1}^{*}-(-1)^{n} \overline{F_{2}}+\overline{F_{3}}-\right.\right.\right.$

$g * k e \alpha \mu m 2+n 2 \pi 2 h 2 t d t-T 0 \alpha \cos n \pi z h+\emptyset 2 s 0(k 1, k 2, \mu m b) c m$

(17)

\section{Determintion Of Thermoelastic Displacement}

From (1) and (16), it gets

$U=\frac{2 \alpha}{h}(1+v) a_{t} \sum_{m=1}^{\infty}\left\{\sum_{n=1}^{\infty} e^{-\alpha\left(\mu_{m}^{2}+\frac{n^{2} \pi^{2}}{h^{2}}\right) t}\left[\int\left\langle\frac{\xi}{k_{1}} s_{0}^{2}\left(k_{1}, k_{2}, \mu_{m} \xi\right) \frac{Q_{0}}{\lambda} f^{*}+\frac{a}{k_{2}} s_{0}^{2}\left(k_{1}, k_{2}, \mu_{m} a\right) F_{1}^{*}-(-1)^{n} \overline{F_{2}}+\right.\right.\right.$

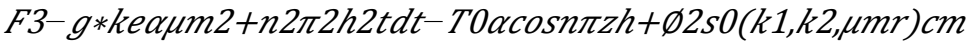

(18)

\section{Determination Of Stress Functions}

Using (18) in (9) and (10), it gets

$\sigma_{r r}=$

$\frac{-4 \mu \alpha}{r h}(1+v) a_{t} \times$

$\sum_{m=1}^{\infty}\left\{\left\langle\frac{\phi^{\prime}}{2}+\sum_{n=1}^{\infty} e^{-\alpha\left(\mu_{m}^{2}+\frac{n^{2} \pi^{2}}{h^{2}}\right) t} \chi^{\prime} \cos \left(\frac{n \pi z}{h}\right)\right\rangle \frac{s_{0}\left(k_{1}, k_{2}, \mu_{m} r\right)}{c_{m}}+\left\langle\frac{\phi}{2}+\sum_{n=1}^{\infty} e^{-\alpha\left(\mu_{m}^{2}+\frac{n^{2} \pi^{2}}{h^{2}}\right) t} \chi \cos \left(\frac{n \pi z}{h}\right)\right\rangle \frac{s_{0}^{\prime}\left(k_{1}, k_{2}, \mu_{m} r\right)}{c_{m}}\right\}$

$\sigma_{\theta \theta}=$

$\frac{-4 \mu \alpha}{h}(1+v) a_{t} \times$

$\sum_{m=1}^{\infty}\left\{\left\langle\frac{\phi^{\prime \prime}}{2}+\sum_{n=1}^{\infty} e^{-\alpha\left(\mu_{m}^{2}+\frac{n^{2} \pi^{2}}{h^{2}}\right) t} \chi^{\prime \prime} \cos \left(\frac{n \pi z}{h}\right)\right\rangle \frac{s_{0}\left(k_{1}, k_{2}, \mu_{m} r\right)}{c_{m}}+\right.$

$2 \emptyset^{\prime} 2+n=100 e-\alpha \mu m 2+n 2 \pi 2 h 2 t \chi^{\prime} \cos n \pi z h s O^{\prime}(k 1, k 2, \mu m r) c m+\phi 2+n=100-\alpha \mu m 2+n 2 \pi 2 h 2 t \chi \cos n \pi z h s O^{\prime \prime}$ $(k 1, k 2, \mu \mathrm{mr}) \mathrm{cm}$

Where

$\chi=\int\left\langle\frac{\xi}{k_{1}} s_{0}^{2}\left(k_{1}, k_{2}, \mu_{m} \xi\right) \frac{Q_{0}}{\lambda} f^{*}+\frac{a}{k_{2}} s_{0}^{2}\left(k_{1}, k_{2}, \mu_{m} a\right) F_{1}^{*}-(-1)^{n} \overline{F_{2}}+\overline{F_{3}}-\frac{\bar{g}^{*}}{k}\right\rangle e^{\alpha\left(\mu_{m}^{2}+\frac{n^{2} \pi^{2}}{h^{2}}\right) t} d t-\frac{T_{0}}{\alpha}$

Set $f=t e^{-t} z(z-h)\left(k_{1}+\xi\right)$

VI. Special Case

And $g=\delta\left(r-r_{0}\right) \delta\left(t-t_{0}\right)$ 
applying Fourier cosine transform to equation (21) and firstly apply finite Marchi-Zgrablich transform to $(22)$ and then Fourier cosine transform it gets

$$
\begin{aligned}
f^{*} & =\frac{h^{3}}{n^{2} \pi^{2}}\left[1+(-1)^{n}\right] t e^{-t}\left(k_{1}+\xi\right) \\
\bar{g}^{*} & =\gamma
\end{aligned}
$$

Substituting the values from (23) and (24) in (16) to (20) it gets

$T=$

$\frac{-2 \alpha}{h} \sum_{m=1}^{\infty}\left\{\sum_{n=1}^{\infty} e^{-\alpha\left(\mu_{m}^{2}+\frac{n^{2} \pi^{2}}{h^{2}}\right) t}\left[\int\left\langle\frac{Q_{0}}{\lambda} \frac{\xi}{k_{1}} s_{0}^{2}\left(k_{1}, k_{2}, \mu_{m} \xi\right) \frac{h^{3}}{n^{2} \pi^{2}}\left[1+(-1)^{n}\right] t e^{-t}\left(k_{1}+\xi\right)+\right.\right.\right.$

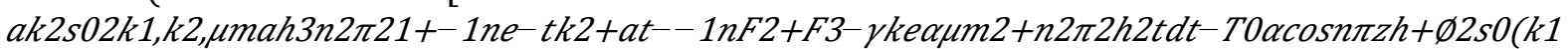
$, k 2, \mu m r) c m$

Where

$\emptyset=e^{-\alpha \mu_{m}^{2} t}\left[\int\left\langle-\frac{Q_{0}}{\lambda} \frac{\xi}{k_{1}} s_{0}^{2}\left(k_{1}, k_{2}, \mu_{m} \xi\right) \frac{h^{3}}{6} t e^{-t}\left(k_{1}+\xi\right)-\frac{a}{k_{2}} s_{0}^{2}\left(k_{1}, k_{2}, \mu_{m} a\right) \frac{h^{3}}{6} t e^{-t}\left(k_{2}+a\right)-\overline{F_{2}}+\overline{F_{3}}-\right.\right.$

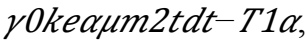

$T_{1}=\left\{\alpha \int\left\langle-\frac{Q_{0}}{\lambda} \frac{\xi}{k_{1}} s_{0}^{2}\left(k_{1}, k_{2}, \mu_{m} \xi\right) \frac{h^{3}}{6} t e^{-t}\left(k_{1}+\xi\right)-\frac{a}{k_{2}} s_{0}^{2}\left(k_{1}, k_{2}, \mu_{m} a\right) \frac{h^{3}}{6} t e^{-t}\left(k_{2}+a\right)-\overline{F_{2}}+\overline{F_{3}}-\right.\right.$ rokeaum2tdtt=0,

$\gamma_{0}=(\gamma)_{n=0}$

$T_{0}=$

$\left[\alpha \int\left\langle\frac{Q_{0}}{\lambda} \frac{\xi}{k_{1}} s_{0}^{2}\left(k_{1}, k_{2}, \mu_{m} \xi\right) \frac{h^{3}}{n^{2} \pi^{2}}\left[1+(-1)^{n}\right] t e^{-t}\left(k_{1}+\xi\right)+\frac{a}{k_{2}} s_{0}^{2}\left(k_{1}, k_{2}, \mu_{m} a\right) \frac{h^{3}}{n^{2} \pi^{2}}\left[1+(-1)^{n}\right] t e^{-t}\left(k_{2}+\right.\right.\right.$ $a--1 n F 2+F 3-\gamma k e \alpha \mu m 2+n 2 \pi 2 h 2 t d t t=0$

$G=$

$\frac{-2 \alpha}{h} \sum_{m=1}^{\infty}\left\{\sum_{n=1}^{\infty} e^{-\alpha\left(\mu_{m}^{2}+\frac{n^{2} \pi^{2}}{h^{2}}\right) t}\left[\int\left\langle\frac{Q_{0}}{\lambda} \frac{\xi}{k_{1}} s_{0}^{2}\left(k_{1}, k_{2}, \mu_{m} \xi\right) \frac{h^{3}}{n^{2} \pi^{2}}\left[1+(-1)^{n}\right] t e^{-t}\left(k_{1}+\xi\right)+\right.\right.\right.$

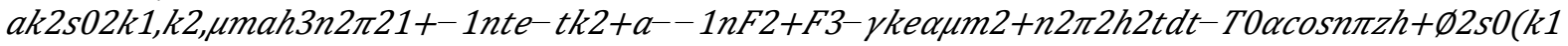
$, k 2, \mu \mathrm{mb}) \mathrm{cm}$

$U=\frac{2 \alpha}{h}(1+v) a_{t} \times \sum_{m=1}^{\infty}\left\{\sum_{n=1}^{\infty} e^{-\alpha\left(\mu_{m}^{2}+\frac{n^{2} \pi^{2}}{h^{2}}\right) t}\left[\int\left\langle\frac{Q_{0}}{\lambda} \frac{\xi}{k_{1}} s_{0}^{2}\left(k_{1}, k_{2}, \mu_{m} \xi\right) \frac{h^{3}}{n^{2} \pi^{2}}\left[1+(-1)^{n}\right] t e^{-t}\left(k_{1}+\xi\right)+\right.\right.\right.$

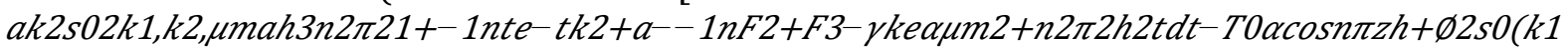
,k2, $\mu \mathrm{mr}) \mathrm{cm}$ 
$\frac{\sigma_{r r}}{r h}(1+v) a_{t} \times$

$\sum_{m=1}^{\infty}\left\{\left\langle\frac{\emptyset^{\prime}}{2}+\sum_{n=1}^{\infty} e^{-\alpha\left(\mu_{m}^{2}+\frac{n^{2} \pi^{2}}{h^{2}}\right) t} \chi^{\prime} \cos \left(\frac{n \pi z}{h}\right)\right\rangle \frac{s_{0}\left(k_{1}, k_{2}, \mu_{m} r\right)}{c_{m}}+\left\langle\frac{\phi}{2}+\sum_{n=1}^{\infty} e^{-\alpha\left(\mu_{m}^{2}+\frac{n^{2} \pi^{2}}{h^{2}}\right) t} \chi \cos \left(\frac{n \pi z}{h}\right)\right\rangle \frac{s_{0}^{\prime}\left(k_{1}, k_{2}, \mu_{m} r\right)}{c_{m}}\right\}$

$\sigma_{\theta \theta}=$

$\frac{-4 \mu \alpha}{h}(1+v) a_{t} \times$

$\sum_{m=1}^{\infty}\left\{\left\langle\frac{\phi^{\prime \prime}}{2}+\sum_{n=1}^{\infty} e^{-\alpha\left(\mu_{m}^{2}+\frac{n^{2} \pi^{2}}{h^{2}}\right) t} \chi^{\prime \prime} \cos \left(\frac{n \pi z}{h}\right)\right\rangle \frac{s_{0}\left(k_{1}, k_{2}, \mu_{m} r\right)}{c_{m}}+\right.$

$2 \varnothing^{\prime} 2+n=1 \infty e-\alpha \mu m 2+n 2 \pi 2 h 2 t \chi^{\prime} \cos n \pi z h s O^{\prime}(k 1, k 2, \mu m r) c m+\phi 2+n=1 \infty e-\alpha \mu m 2+n 2 \pi 2 h 2 t \chi \cos n \pi z h s 0^{\prime \prime}$ $(k 1, k 2, \mu m r) c m$

Where

$\chi=\int\left\langle\frac{Q_{0}}{\lambda} \frac{\xi}{k_{1}} s_{0}^{2}\left(k_{1}, k_{2}, \mu_{m} \xi\right) \frac{h^{3}}{n^{2} \pi^{2}}\left[1+(-1)^{n}\right] t e^{-t}\left(k_{1}+\xi\right)+\frac{a}{k_{2}} s_{0}^{2}\left(k_{1}, k_{2}, \mu_{m} a\right) \frac{h^{3}}{n^{2} \pi^{2}}\left[1+(-1)^{n}\right] t e^{-t}\left(k_{2}+\right.\right.$ $a--1 n F 2+F 3-\gamma k e \alpha \mu m 2+n 2 \pi 2 h 2 t d t-T 0 \alpha$ and

$\overline{F_{2}}=e^{-t} h t \int_{a}^{\xi} r^{2} s_{0}\left(k_{1}, k_{2}, \mu_{m} r\right) d r, \overline{F_{3}}=-e^{-t} h t \int_{a}^{\xi} r^{2} s_{0}\left(k_{1}, k_{2}, \mu_{m} r\right) d r$

\section{Numerical Results}

Take $a=1 \mathrm{~cm}, b=2 \mathrm{~cm}, \xi=1.5 \mathrm{~cm}, h=0.5 \mathrm{~cm}, k=0.0141$

$G=-4 \alpha \sum_{m=1}^{\infty}\left\{\sum_{n=1}^{\infty} e^{-\alpha\left(\mu_{m}^{2}+39.43 n^{2}\right) t}\left[\int\left\langle\frac{Q_{0}}{\lambda} \frac{1.5}{k_{1}} s_{0}^{2}\left(k_{1}, k_{2}, 1.5 \mu_{m}\right) \frac{0.012}{n^{2}}\left[1+(-1)^{n}\right] e^{-t}\left(k_{1}+1.5\right)+\right.\right.\right.$

$1 \mathrm{k} 2 \mathrm{~s} 02 \mathrm{k} 1, \mathrm{k} 2, \mu \mathrm{m} 0.012 \mathrm{n} 21+-1 \mathrm{ne}-\mathrm{tk} 2+1--1 \mathrm{nF} 2-+\mathrm{F3}-70.92 \mathrm{\gamma e \alpha um} 2+39.43 \mathrm{n} 2 \mathrm{tdt}-\mathrm{TO} \alpha \cos 6.28 \mathrm{nz}+\varnothing 2$ $\mathrm{sO}(\mathrm{k1}, \mathrm{k2}, 2 \mu \mathrm{m}) \mathrm{cm}$

Where

$\emptyset=e^{-\alpha \mu_{m}^{2} t}\left[\int\left\langle-\frac{Q_{0}}{\lambda} \frac{1.5}{k_{1}} s_{0}^{2}\left(k_{1}, k_{2}, 1.5 \mu_{m}\right)(0.0208) t e^{-t}\left(k_{1}+1.5\right)-\frac{a}{k_{2}} s_{0}^{2}\left(k_{1}, k_{2}, \mu_{m}\right)(0.0208) t e^{-t}\left(k_{2}+1\right)-\right.\right.$

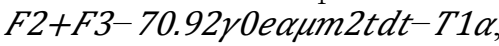

$T_{1}=\left\{\alpha \int\left\langle-\frac{Q_{0}}{\lambda} \frac{1.5}{k_{1}} s_{0}^{2}\left(k_{1}, k_{2}, 1.5 \mu_{m}\right)(0.0208) t e^{-t}\left(k_{1}+1.5\right)-\frac{a}{k_{2}} s_{0}^{2}\left(k_{1}, k_{2}, \mu_{m}\right)(0.0208) t e^{-t}\left(k_{2}+1\right)-\overline{F_{2}}+\right.\right.$

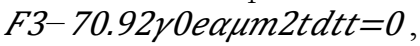

$\gamma_{0}=(\gamma)_{n=0}, \overline{F_{2}}=0.5 e^{-t} t \int_{a}^{\xi} r^{2} s_{0}\left(k_{1}, k_{2}, \mu_{m} r\right) d r, \overline{F_{3}}=-0.5 e^{-t} t \int_{a}^{\xi} r^{2} s_{0}\left(k_{1}, k_{2}, \mu_{m} r\right) d r$

AND

$T_{0}=$

$\left[\alpha \int\left\langle\frac{Q_{0}}{\lambda} \frac{1.5}{k_{1}} s_{0}^{2}\left(k_{1}, k_{2}, 1.5 \mu_{m}\right) \frac{0.012}{n^{2}}\left[1+(-1)^{n}\right] t e^{-t}\left(k_{1}+1.5\right)+\frac{1}{k_{2}} s_{0}^{2}\left(k_{1}, k_{2}, \mu_{m}\right) \frac{0.012}{n^{2}}\left[1+(-1)^{n}\right] t e^{-t}\left(k_{2}+\right.\right.\right.$ $1--1 n F 2+F 3-0.5 t e-t r-70.92 \gamma e \alpha \mu m 2+39.43 n 2 t d t t=0$

\section{Conclusions}

In this paper temperature distribution, thermoelastic displacement and stress functions are determined by using finite Marchi-Zgrablich transform and Fourier cosine transform for an annular disc with the effect of partially distributed heat supply and internal heat generation. The results are obtained in the form of infinite series and Bessel's function. 


\section{References}

[1] Khobragade N.W. and Lamba N.K.: "Thermal Stresses of a Thin Annular Disc Due to Partially Distributed Heat Supply", Int. J Latest Trend Math Vol-1 No. 1 October 2011

[2] Marchi E and Zgrablich G: Heat conduction in Hollow cylinder with radiation Proc. Edingburgh Math. Soc. V1.14 Part 2(1964), pp159-164.

[3] N.M.Ozisik: Boundary value problem of heat conduction, International text book co. Scranton, Pennsylvania, (1968) pp. 481-492

[4] Nowacki, W.: "The state of stress in a thick circular plate due to a temperature field", Bull. Acad. Polon. Sci. ser., Tech., Vol. IV, No.5, 1957.

[5] Roychoudhary S. K.: "A note on the quasi-static thermal stresses in a thin circular plate due to transient temperature applied along the circumferences of a circle over the upper face", Bull. Acad. Polon. Sci. ser., Tech. No. 1, pp.20-21, 1972.

[6] Wankhede P.C: “On the Quasi-static thermal stresses in a circular plate,” Indian J.pur and Appl. Math. 13(11), pp. 1273-1277, 1982. 